the polyclonal activation of B cells, leading to the synthesis of a large variety of autoantibodies.

Objectives: The aim of this study is to describe the prevalence of atypical autoantibodies in patients with pSS from the SjogrenSER registry.

Methods: SjogrenSER registry is a multicenter transversal study of pSS patients fulfilling European/American consensus criteria 2002. Patients were included by randomization from thirty-three Rheumatology Spanish departments. Data were collected by reviewing clinical records and interviewing the patients. Informed consent was obtained and local ethics committees approved the study. Variables were analysed by descriptive statistical methods, using means, medians, and rates. Chi-square was used to establish the statistical associations, being considered a $\mathrm{p}<0,05$ as significant.

Results: Four hundred and thirty-seven patients were included. Ninety-five percent of them were women. The median age of the cohort was 58 years. Twenty-three patients had AntiDNA (5.26\%), 10 patients AntiSm (2.29\%), 23 patients AntiRNP (5.26\%) and 26 patients antiphospholipid antibodies (5.95\%). Regarding AntiDNA+ patients, there were minimal non-significant differences in age at diagnosis and age at onset of symptoms compared to AntiDNA- patients ( 47 vs 50.5 years and 43.5 vs 46.5 years, respectively). The association with some systemic manifestation was only observed with joint involvement, which was significantly more frequent in AntiDNA+ patients ( $56.5 \%$ vs $34.2 \%, p=0.031$ ). Regarding AntiSm+ patients, a significant negative association with AntiDNA antibodies was observed, being $70 \%$ of patients AntiDNA-; we also found a significant positive association with AntiRo and AntiLa, being $100 \%$ and $68 \%$ of patients AntiRo+ and AntiLa+ respectively. A significant negative association with lymphopenia was observed (no AntiSm+ patient had lymphopenia). AntiRNP+ patients showed a significant negative association with AntiDNA antibodies, being $80 \%$ of patients AntiDNA-, and a significant positive association with AntiRo, being $96 \%$ patients AntiRo+. A significant positive association was also observed with decreased C4 compared to AntiRNP- patients ( $28 \%$ vs $13.38 \%$, $\mathrm{p}=0.025$ ). Regarding patients with antiphospholipid antibodies, a significant negative association was observed with antiDNA antibodies, being $93 \%$ of patients AntiDNA-. A significant positive association with some systemic manifestation was only observed with the presence of anemia (44\% vs $17.7 \%$ ). A significant positive association with decreased $\mathrm{C} 3$ and $\mathrm{C} 4$ was also observed, compared with the AntiRNP- patients (C3 $20 \%$ vs $13.67 \%$ and C4 33\% vs $12.67 \%$ ).

Conclusions: More than $5 \%$ of pSS patients had antibodies characteristic of other autoimmune diseases. These atypical autoantibodies were significantly related to some pSS characteristic: antiSm, antiRNP and antiphospholipid antibodies were significantly related to the presence of antiRo, antiDNA antibodies were significantly related to joint involvement, antiphospholipid antibodies were significantly related to anemia, and antiRNP and antiphospholipid antibodies were significantly related to hypocomplementemia.

Disclosure of Interest: None declared

DOI: 10.1136/annrheumdis-2017-eular.2600

\section{SAT0300 HIGH PROPORTIONS OF DEMENTIA AMONG SLE PATIENTS: A BIG DATA ANALYSIS}

O. Gendelman ${ }^{1,2}$, S. Tiosano ${ }^{1,2}$, D. Comaneshter ${ }^{3}$, A. Cohen ${ }^{3,4}$, Y. Shoenfeld ${ }^{1,2}, \mathrm{H}$. Amital ${ }^{1,2}$. ${ }^{1}$ Internal Medicine 'B', Zabludowicz Center for Autoimmune Diseases, Sheba Medical Center, Ramat-Gan; ${ }^{2}$ Sackler School of Medicine, Tel-Aviv University; ${ }^{3}$ Chief physician's office, Clalit Health Services, Tel-Aviv; ${ }^{4}$ Siaal Research Center for Family Medicine and Primary Care, Faculty of Health Sciences, Ben Gurion University of the Negev, Beer Sheva, Israel

Background: Systemic lupus erythematosus (SLE) is a chronic autoimmune disease affecting wide range of systems including the peripheral and central nervous system ${ }^{1}$. Cognitive impairment leading to dementia is one of the harmful central nervous system manifestations of SLE ${ }^{2}$

Objectives: The aim of this study was to investigate the association of SLE and dementia.

Methods: A cross-sectional study was performed utilizing Clalit Health Care database, the largest $\mathrm{HMO}$ in Israel with more than 4.4 million enrollees. SLE patients were compared in a 1:5 ratio to age and sex matched controls. Chi-square and $t$ tests were used for univariate analysis, and a logistic regression model was used for multivariate analysis.

Results: The study included 4886 SLE patients and 24,430 age and sex frequency matched controls without SLE. The proportion of dementia was higher among SLE patients compared to controls $(1.56 \%$ and $0.51 \%$ respectively; $\mathrm{p}<0.001)$. This finding was consistent across all age groups by univariate analysis. In a multivariate logistic regression analysis, SLE was significantly associated with dementia $(\mathrm{OR}=2.039,95 \% \mathrm{Cl}=1.110-2.039$ ).

Conclusions: SLE is significantly associated with dementia. This finding should give rise to search for SLE in patients with an ambiguous cause for dementia, especially those with an early onset cognitive decline.

\section{References:}

[1] Tsokos GC. Systemic Lupus Erythematosus. N Engl J Med. 2011;365(22): 2110-2121.

[2] Liang MH, Corzillius M, Bae SC, et al. The American College of Rheumatology nomenclature and case definitions for neuropsychiatric lupus syndromes. Arthritis Rheum. 1999;42(4):599-608.

Disclosure of Interest: None declared
DOI: 10.1136/annrheumdis-2017-eular.5245

\section{SAT0301 SERUM AUTOANTIBODY PROFILING OF PRIMARY SJÖGREN'S SYNDROME PATIENTS REVEALS NOVEL BIOMARKERS ASSOCIATED WITH THE DISEASE, DISEASE ACTIVITY, AND CLINICAL RESPONSE TO VAY736}

P. Budde ${ }^{1}$, J. Doucet ${ }^{2}$, H.-D. Zucht ${ }^{1}$, R. Kazma ${ }^{2}$, P. Maguire ${ }^{2}$, A. Avrameas ${ }^{2}$, M.-A. Valentin ${ }^{2}$, S. Oliver ${ }^{3}$, P. Schulz-Knappe ${ }^{1}$, A. Vitaliti ${ }^{2} .{ }^{1}$ Medical Research, Protagen, Dortmund, Germany; ${ }^{2}$ Translational Medicine/Biomarker

Development; ${ }^{3}$ Translational Medicine, Novartis Institutes for Biomedical Research, Basel, Switzerland

Background: Overexpression of B cell activating factor (BAFF) in salivary glands contributes to the pathogenesis of primary Sjögren's syndrome (pSS) by promoting autoantibody (AAB) production. Treatment of pSS patients with VAY736, an anti-BAFF receptor mAb, appears promising and was associated with a depletion of circulating $B$ cells and a positive therapeutic effect [1]. In addition to the classical anti-SS-A/Ro and anti-SS-B/La, a broader set of AABs may reflect $B$ cell disturbances in pSS and could serve as markers during clinical development of novel pSS therapeutics.

Objectives: In this study, we explored novel AABs in pSS patients and healthy controls (HCs) and we tested their associations with the disease, disease activity, and clinical response to VAY736.

Methods: Reactivity of AABs to 1,596 antigens was measured in serum samples from 27 pSS patients from a placebo-controlled trial at baseline and posttreatment week 12 and from 50 age and gender-matched HCs. Patients were treated at baseline with a single dose of VAY736 at $10 \mathrm{mg} / \mathrm{kg}(\mathrm{n}=12), 3 \mathrm{mg} / \mathrm{kg}$ $(n=6)$, or placebo $(n=9)$. First, to identify AABs associated with pSS, 3 different methods compared $A A B$ levels at baseline between pSS patients and HCs: Wilcoxon rank sum test, significance analysis of microarrays, and comparison of the 90th quantiles between groups. Second, to identify AABs associated with pSS activity, Pearson correlation of AABs with EULAR Sjögren's Syndrome Disease Activity Index, secondary outcomes, and salivary and serum BAFF were tested, using baseline and week 12 levels as well as relative changes. Third, VAY736 treatment-specific changes in AAB levels were investigated using linear mixed-effects models adjusting for dosage, age, and gender effects.

Results: Of 1,596 antigens, 36 were statistically different between pSS patients and $\mathrm{HCs}$ for at least one of the 3 tests, including the known SS-A/Ro and SS-B/La (significant for all 3 tests) as well as novel antigens. SS-A/Ro and SS-B/La AABs were not associated with disease activity or response to treatment. However, 48 AABs were significantly correlated with pSS activity combining all treatment arms, and 12 AABs had baseline values that correlated with change in pSS activity upon VAY736 treatment (unadjusted $\mathrm{p}<0.05$ ). Interestingly, 51 serum AABs correlated with BAFF saliva levels $(|r|>0.55)$, but not with BAFF serum levels. The genes encoding novel antigens are involved in apoptotic, anti-viral, metabolic, inflammatory, blood coagulation and B-cell processes, suggesting a possible link to the disease pathology.

Finally, there was no reduction in AABs levels in response to VAY736, possibly because the 12 weeks post-treatment analysis was too short to identify large effects.

Conclusions: In conclusion, we identified new AABs in pSS patients that have the potential to serve as markers of diagnosis, pSS activity, or as predictors of clinical outcome measures. Further large-scale studies are needed to confirm the value of these markers.

References:

[1] Dörner T et al. Arthritis Rheum 2016; 68(suppl S10):4051.

Disclosure of Interest: P. Budde Employee of: Protagen AG, J. Doucet Employee of: Novartis AG, H.-D. Zucht Employee of: Protagen AG, R. Kazma Employee of: Novartis AG, P. Maguire Employee of: Novartis AG, A. Avrameas Employee of: Novartis AG, M.-A. Valentin Employee of: Novartis AG, S. Oliver Employee of: Novartis AG, P. Schulz-Knappe Employee of: Protagen AG, A. Vitaliti Employee of: Novartis AG

DOI: 10.1136/annrheumdis-2017-eular.1905

\section{SAT0302 ANALYSIS OF 9302 PATIENTS FROM THE BIG DATA INTERNATIONAL PRIMARY SJÖGREN SYNDROME COHORT: CLINICAL PRESENTATION AT DIAGNOSIS OF EUROPEAN VS NON-EUROPEAN PATIENTS}

P. Brito-Zerón $^{1,2}$, N. Acar-Denizli ${ }^{3}$, M. Zeher ${ }^{4}$, A. Rasmussen ${ }^{5}$, R. Seror ${ }^{6}$ T. Mandl ${ }^{7}$, X. Li ${ }^{8}$, C. Baldini ${ }^{9}$, J.-E. Gottenberg ${ }^{10}$, D. Danda ${ }^{11}$, R. Priori ${ }^{12}$ L. Quartuccio ${ }^{13}$, G. Hernandez-Molina ${ }^{14}$, A.A. Kruize ${ }^{15}$, S.-H. Park ${ }^{16}$,

M. Kvarnström ${ }^{17}$, S. Praprotnik ${ }^{18}$, D. Sene ${ }^{19}$, E. Bartoloni ${ }^{20}$, R. Solans ${ }^{21}$, Y. Suzuki ${ }^{22}$, D. Isenberg ${ }^{23}$, M. Rischmueller ${ }^{24}$, G. Nordmark ${ }^{25}$, G. Fraile ${ }^{26}$, A. Sebastian ${ }^{27}$, A. Vissink ${ }^{28}$, T. Nakamura ${ }^{29}$, V. Valim ${ }^{30}$, R. Giacomelli ${ }^{31}$ V. Devauchelle-Pensec ${ }^{32}$, B. Hofauer ${ }^{33}$, M. Bombardieri ${ }^{34}$, V. Trevisani ${ }^{35}$, D. Hammenfors ${ }^{36}$, S.E. Carsons ${ }^{37}$, S.G. Pasoto ${ }^{38}$, J. Morel ${ }^{39}$, S. Retamozo ${ }^{40}$ T.A. Gheita ${ }^{41}$, F. Atzeni ${ }^{42}$, C. Vollenveider ${ }^{43}$, X. Mariette ${ }^{6}$, M. Ramos-Casals ${ }^{2}$ on behalf of the EULAR-SS Task Force Big Data Consortium. ${ }^{1}$ Hosp CIMA-Sanitas; ${ }^{2}$ Hosp Clínic, Barcelona, Spain; ${ }^{3}$ Msgsü, Istanbul, Turkey; ${ }^{4}$ Univ, Debrecen, Hungary; ${ }^{5}$ OMRF, Oklahoma, United States; ${ }^{6}$ Univ Paris Sud, Paris, France; ${ }^{7}$ Lund Univ, Malmö, Sweden, ${ }^{8}$ Anhui Hosp, Hefei, China; ${ }^{9}$ Univ, Pisa, 